\title{
Bioinformatics analysis of key differentially expressed genes in well and poorly differentiated endometrial carcinoma
}

\author{
YUQIN ZANG, MENGTING DONG, KAI ZHANG, WENYAN TIAN, YINGMEI WANG and FENGXIA XUE \\ Department of Gynecology and Obstetrics, Tianjin Medical University General Hospital, Tianjin 300052, P.R. China
}

Received October 25, 2017; Accepted April 26, 2018

DOI: $10.3892 / \mathrm{mmr} .2018 .8969$

\begin{abstract}
Endometrial carcinoma (EC) is one of the most common gynecological malignancies. The malignant degree increases between grade (G)1 and G3, and EC of G3 usually presents a high recurrence rate and poor prognosis. Therefore, the present study aimed to examine the principal genes associated with the degree of differentiation in EC. The microarrays GSE17025, GSE24537 and GSE35784, representing data of Type I EC samples of G1 and G3, were downloaded from the Gene Expression Omnibus. The differentially expressed genes (DEGs) and differentially expressed micro (mi)RNAs (DEMs) were identified, followed by functional enrichment analyses and interaction network construction. In total, 83 upregulated and 130 downregulated DEGs with the same expression trends in two mRNA datasets were screened. The upregulated DEGs were primarily enriched in 'mitotic cell cycle process', 'cell cycle process' and 'mitotic cell cycle'; while the downregulated DEGs were enriched in 'cellular component assembly involved in morphogenesis', 'cell projection organization' and 'microtubule-based movement'. From the protein-protein interaction network, DNA topoisomerase II $\alpha$, kinesin family member 11, cyclin B1 and BUB1 mitotic checkpoint serine/threonine were identified as foremost hub genes. One module was extracted and involved in 'mitotic cell cycle process' and 'cell cycle process'. Based on the analysis of DEMs and the miRNA-target regulatory network, miRNA-9 may be the most important upregulated DEM, and the DEGs forkhead box P1 and cyclin E1 may serve vital roles in the differentiation of EC. In conclusion, principal genes were identified that may be determinants of the
\end{abstract}

Correspondence to: Dr Yingmei Wang or Dr Fengxia Xue, Department of Gynecology and Obstetrics, Tianjin Medical University General Hospital, 154 Anshan Road, Heping, Tianjin 300052, P.R. China

E-mail: wangyingmei1978@126.com

E-mail: fengxiaxue1962@gmail.com

Key words: endometrial carcinoma, bioinformatics analysis, differentially expressed genes, functional enrichment analysis, protein-protein interaction network, microRNA-target regulatory network carcinogenesis of poorly differentiated EC, which may facilitate the examination of potential molecular mechanisms. These genes may additionally help identify candidate biomarkers and novel therapeutic targets for poorly differentiated EC.

\section{Introduction}

Endometrial carcinoma (EC) is one of the most common female reproductive tract malignancies globally (1-5). Type I EC, additionally termed endometrioid endometrial carcinoma (EEC) or endometrioid adenocarcinoma (EAC), accounts for $80-90 \%$ of all subtypes of EC (6). Based on the degree of differentiation, EEC is classified into three grades: G1 (well-differentiated EEC), G2 (moderately differentiated EEC) and G3 (poorly differentiated EEC). The diagnosis of the EEC grade primarily depends on the histopathological examination of the structure of tumor tissue and/or nuclear atypia. The higher the grade, the more malignant the behavior of the tumor. Poorly differentiated EEC usually develops rapidly and presents with metastasis in a short time. A previous study demonstrated that lymph vascular space invasion, which is an important factor in prognostic evaluation and therapeutic strategy determination, is uncommon in EEC of G1 and G2; however, it is common in EEC of G3 (7). Deep myometrial infiltration is additionally common in EEC of G3. Thus, the treatment of different grades of EEC varies. For patients with EEC of G1 with $<50 \%$ myometrial invasion, a systematic lymphadenopathy bares no benefit; however, for patients with EEC of G3, lymphadenectomy is considered or should be recommended (8). In addition, adjuvant chemotherapy, radiotherapy or hormone therapy is necessary for EEC of G3. Due to the unfavorable biological behavior, the recurrence of G3 EEC is high. Therefore, it is important to investigate the molecular mechanism underlying how poorly differentiated EEC occurs or develops, and to identify novel targeted therapy. As mentioned above, the present study aimed to reveal the difference between EEC of G1 and G3 at the gene level, and to identify candidate biomarkers or therapeutic targets for EEC of $\mathrm{G} 3$.

Bioinformatics analysis of gene microarrays is a popular method to detect the expression of mRNA alterations between different samples on a high-throughput platform (9). It is efficient to screen the differentially expressed genes (DEGs) once among a mass of data and furthermore, function, pathway and interaction analyses regarding the genes may be implemented. 
Additionally, microRNAs (miRNAs), small noncoding single-stranded RNAs, perform vital roles in tumorigenesis by regulating their target genes, and may be used for targeted DEG prediction. It is necessary and meaningful to investigate the molecular alterations in well and poorly differentiated EEC; however, no comprehensive study of bioinformatics analysis has been conducted in this regard, to the best of the authors' knowledge. Consequently, relevant microarrays were searched for in the Gene Expression Omnibus (GEO) database, and a bioinformatics analysis was conducted, including identification of DEGs and differentially expressed miRNAs (DEMs), together with function and pathway enrichment analyses and interaction network construction. From the present study, novel clues regarding the association between gene expression and differentiation degree of EEC may be determined.

\section{Materials and methods}

Microarray data. The gene expression profiles of EEC were obtained from the GEO at the National Center for Biotechnology Information (http://www.ncbi.nlm.nih.gov/geo/), which is an international public repository that allows users to upload or download high-throughput microarrays freely. The mRNA microarray dataset GSE17025 contains 103 samples, among which 79 samples were stage I EECs including 30 samples of G1, 33 samples of G2 and 16 samples of G3. A total of 24 samples were 12 papillary serious carcinoma samples and 12 atrophic endometrium samples, which were subsequently excluded. The other mRNA microarray dataset GSE24537 contains 33 early stage EC samples, including 11 EAC samples of G1, 11 EAC samples of G3 and 11 uterine serous carcinoma samples. The miRNA microarray dataset GSE35784 includes six G1 EEC samples, nine G2 EEC samples, three G3 EEC samples and four normal endometrium samples.

Identification of DEGs. To analyze these data, the online tool GEO2R (https://www.ncbi.nlm.nih.gov/geo/geo2r/) was used. GEO2R is an interactive tool for users to identify genes that are differentially expressed across experimental conditions by comparing two groups of samples in a GEO series. In the present study, 30 G1 EEC samples and 16 G3 EEC samples from the dataset GSE17025 were analyzed with 11 G1 EAC samples and $11 \mathrm{G} 3$ EAC samples from the dataset GSE24537. $\mid \log \mathrm{FCl} \geq 1$ and $\mathrm{P}<0.05$ were set as the cut-off values. Subsequently, the genes, which were differentially expressed with the same expression trends in datasets GSE17025 and GSE24537 were selected.

Gene Ontology (GO) and Kyoto Encyclopedia of Genes and Genomes (KEGG) analyses of DEGs. The GO (http://geneontology.org/) is a popular repository that allows investigators to acquire information on categories and attributes of genes, which contains biological processes (BP), cellular components (CC) and molecular functions (MF) (10). The KEGG database (http://www.genome.jp/) is a knowledge base for the systematic analysis of gene functional and metabolic pathways (11). With the GO and KEGG enrichment analyses, primary information regarding the function and pathways of the identified DEGs was acquired. To perform the analyses, the Database for Annotation, Visualization and
Integrated Discovery (DAVID, http://david.abcc.ncifcrf.gov/) was used, which provides a comprehensive set of functional annotation tools for users to understand the biological meaning of genes (12). $\mathrm{P}<0.05$ was considered to indicate a statistically significant difference.

Protein-protein interaction (PPI) network construction. The PPI network, constructed using the Search Tool for the Retrieval of Interacting Genes (STRING) database (http://string-db.org/), is a visualized molecular interaction network that presents the associations among the proteins encoded through the identified DEGs (13). Cytoscape (http://www.cytoscape.org/) is a software for visualizing the PPI network (14), and the plugin Molecular Complex Detection (MCODE; http://apps.cytoscape.org/apps/mcode) is designed for module analysis. In the present study, STRING (version 10.0) and Cytoscape (version 3.5.0) were used to construct the PPI network with combined score $>0.4$ as the cut-off value. Using NetworkAnalyzer in Cytoscape (http://apps.cytoscape. org/apps/networkanalyzer), the top 10 hub DEGs with the highest node degrees were determined. Using the MCODE, modules of the PPI network were extracted, and only modules with scores $>4$ were selected to receive further function and pathway enrichment analyzes. $\mathrm{P}<0.05$ was considered to indicate a statistically significant difference.

miRNA-DEG pairs. DEMs between G3 and G1 EEC samples of the dataset GSE35784 were identified using GEO2R. $\mid \log \mathrm{FCl} \geq 1$ and P-value $<0.05$ were set as the threshold. The screened DEGs were uploaded to the online tool WebGestalt (http://www.webgestalt.org), and the miRNA-target gene enrichment analysis was performed. WebGestalt is a comprehensive, flexible and interactive gene set enrichment analysis toolkit, from which information about miRNA-target gene interactions may be obtained. Subsequently, the miRNA-target regulatory network was constructed using Cytoscape.

\section{Results}

Identification of the DEGs. The DEGs were identified using GEO2R by comparing the data of well-differentiated samples with the poorly differentiated samples. With the criteria of $\mid \log \mathrm{FCl} \geq 1$ and $\mathrm{P}<0.05$, a total of 1,456 and 1,739 genes were screened as DEGs from the microarray datasets GSE17025 and GSE24537, respectively. Among them, 243 upregulated and 1,213 downregulated genes were identified in GSE17025, while 869 upregulated and 870 downregulated genes were identified in GSE24537. Subsequently, 213 DEGs with the same expression trends in the two datasets were screened, of which 83 genes were upregulated and 130 genes were downregulated. The expression heat maps of the top 100 genes of the matched 213 DEGs are presented in Fig. 1.

$G O$ and KEGG analyses. With the functional annotation tool DAVID Bioinformatics Resources 6.8, the results of GO and KEGG analyses were obtained (Tables I and II). The top five results of $\mathrm{BP}, \mathrm{CC}$ and $\mathrm{MF}$ in $\mathrm{GO}$ analysis with $\mathrm{P}<0.05$ are listed in Table I, which revealed that the upregulated DEGs were primarily involved in 'mitotic cell cycle process', 'cell cycle process', 'mitotic cell cycle', 'cell cycle' and 'chromosome 
A

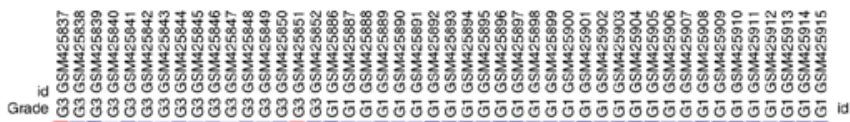

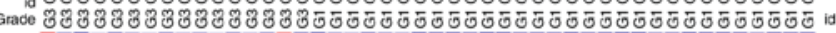
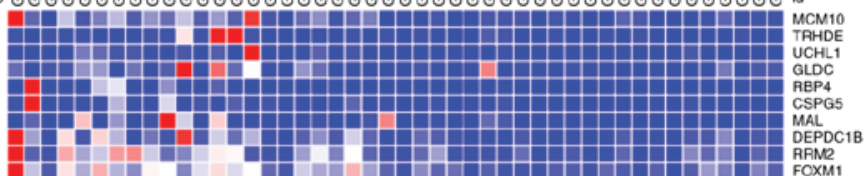

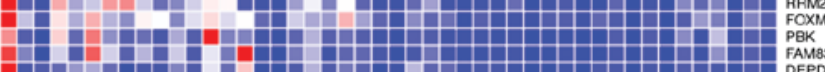
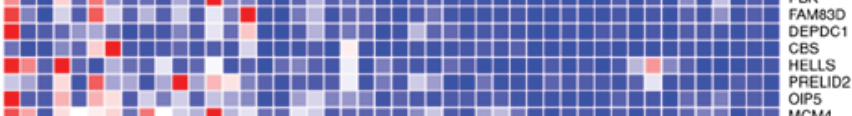

YH -

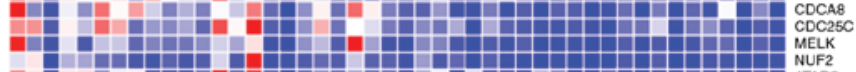

a
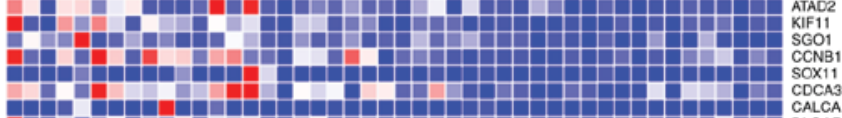

C10

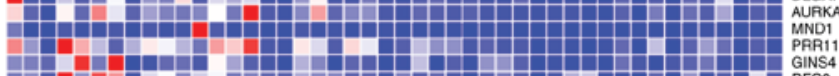

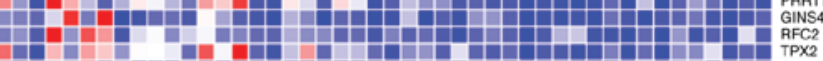
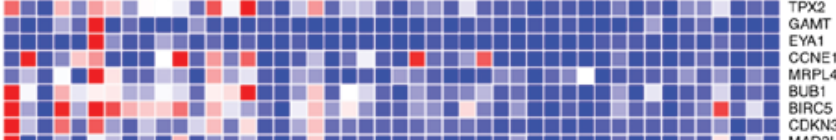
20

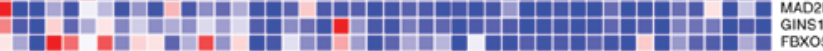
H. - 1 (1)

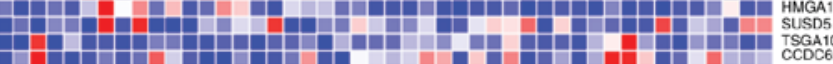

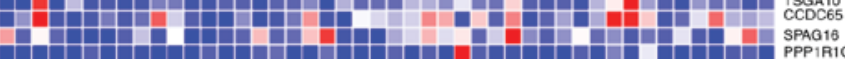

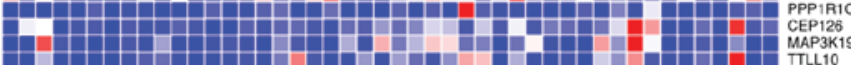

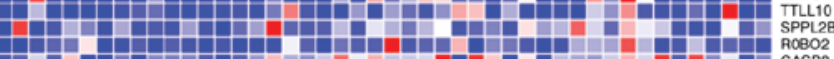

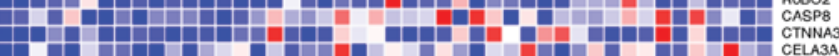

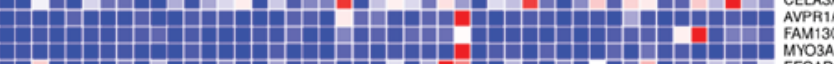

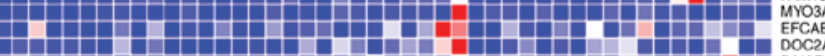

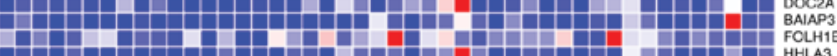
T1

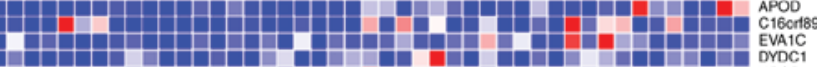

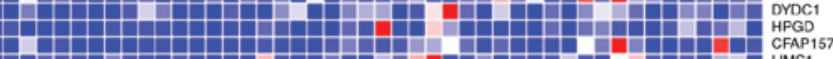

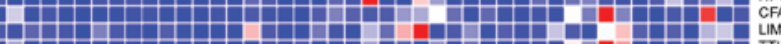

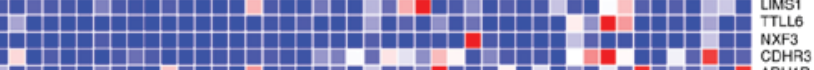

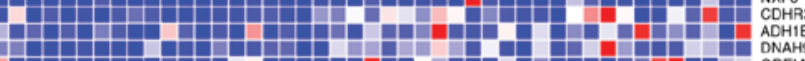

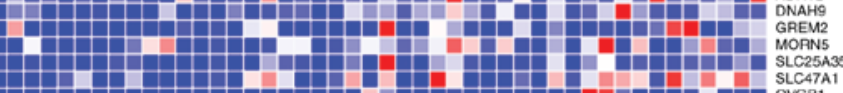

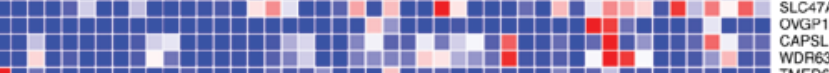

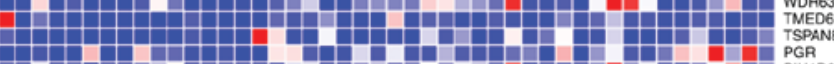

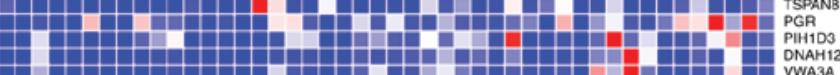

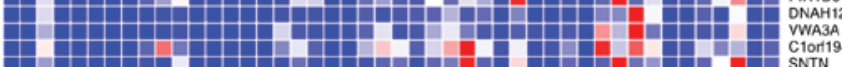

B

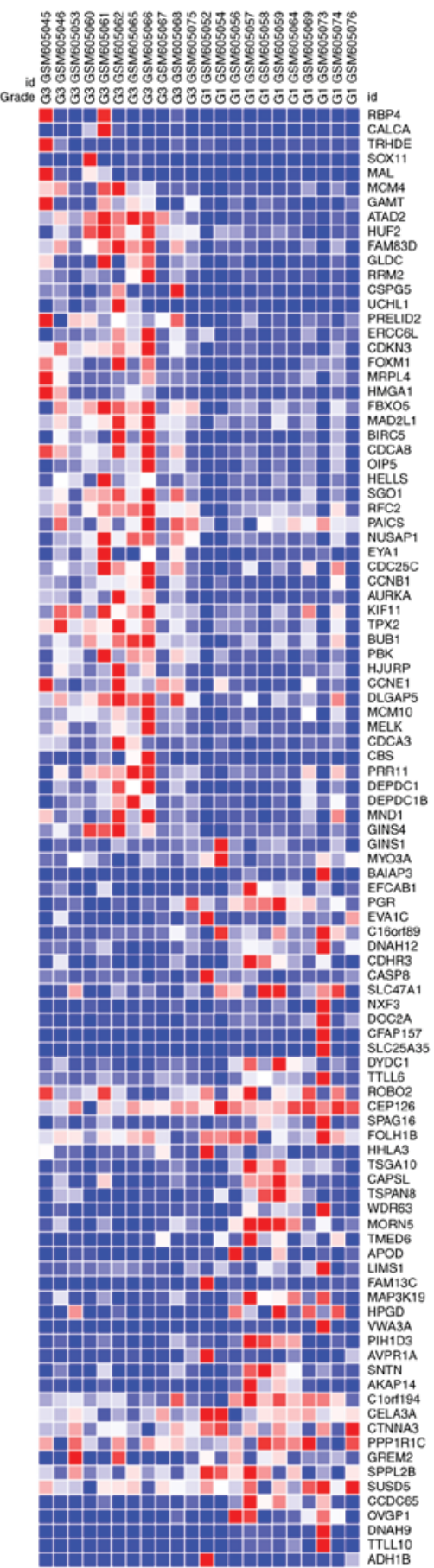

Figure 1. Heat map of the top 100 DEGs (50 upregulated DEGs and 50 downregulated DEGs). (A) Expression heat map of the top DEGs in GSE17025. (B) Expression heat map of the top DEGs in GSE24537. Red, upregulated DEGs; blue, downregulated DEGs. DEGs, differentially expressed genes.

segregation'; while the downregulated DEGs were primarily involved in 'cellular component assembly involved in morphogenesis', 'cell projection organization', 'microtubule-based movement', 'cilium organization' and 'microtubule-based process'. Results of the KEGG pathway enrichment analysis with $\mathrm{P}<0.05$ are presented in Table II. The upregulated DEGs were primarily enriched in 'cell cycle', 'oocyte meiosis' and 'progesterone-mediated oocyte maturation'; while the downregulated DEGs were enriched in 'drug metabolism cytochrome $\mathrm{P} 450$ ', 'metabolism of xenobiotics by cytochrome $\mathrm{P} 450$ ' and 'chemical carcinogenesis'.
PPI network analysis. Using the STRING database and Cytoscape software, the PPI network with 120 nodes and 1,300 edges was constructed. Using the Network Analyzer tool, the top 10 hub DEGs were obtained (Fig. 2). As demonstrated in Fig. 2, the hub genes were DNA topoisomerase II $\alpha$ (TOP2A), kinesin family member 11 (KIF11), cyclin B1 (CCNB1), BUB1 (BUB1 mitotic checkpoint serine/threonine kinase), TTK protein kinase, MAD2 mitotic arrest deficient-like 1 (yeast), NDC80, kinetochore complex component, maternal embryonic leucine zipper kinase, non-SMC condensing I complex subunit $\mathrm{G}$ and aurora kinase A (AURKA). Furthermore, 
Table I. GO analysis of the DEG associated with the degree of differentiation in EEC.

\begin{tabular}{|c|c|c|c|}
\hline Category & Term & Count & P-value \\
\hline \multicolumn{4}{|l|}{ A, upregulated } \\
\hline GOTERM_BP_FAT & GO:1903047 mitotic cell cycle process & 26 & $1.65 \times 10^{20}$ \\
\hline GOTERM_BP_FAT & GO:0022402 cell cycle process & 31 & $6.67 \times 10^{20}$ \\
\hline GOTERM_BP_FAT & GO:0000278 mitotic cell cycle & 26 & $6.32 \times 10^{19}$ \\
\hline GOTERM_BP_FAT & GO:0007049 cell cycle & 31 & $8.78 \times 10^{18}$ \\
\hline GOTERM_BP_FAT & GO:0007059 chromosome segregation & 17 & $1.67 \times 10^{14}$ \\
\hline GOTERM_CC_FAT & GO:0005694 chromosome & 25 & $1.77 \times 10^{14}$ \\
\hline GOTERM_CC_FAT & GO:0000775 chromosome, centromeric region & 14 & $4.87 \times 10^{14}$ \\
\hline GOTERM_CC_FAT & GO:0044427 chromosomal part & 23 & $3.74 \times 10^{13}$ \\
\hline GOTERM_CC_FAT & GO:0005819 spindle & 14 & $1.50 \times 10^{11}$ \\
\hline GOTERM_CC_FAT & GO:0098687 chromosomal region & 15 & $1.84 \times 10^{11}$ \\
\hline GOTERM_MF_FAT & GO:0005524 ATP binding & 14 & $6.46 \times 10^{4}$ \\
\hline GOTERM_MF_FAT & GO:0032559 adenyl ribonucleotide binding & 14 & $7.68 \times 10^{4}$ \\
\hline GOTERM_MF_FAT & GO:0030554 adenyl nucleotide binding & 14 & $7.99 \times 10^{4}$ \\
\hline GOTERM_MF_FAT & GO:0003697 single-stranded DNA binding & 4 & $2.51 \times 10^{3}$ \\
\hline GOTERM_MF_FAT & GO:0035639 purine ribonucleoside triphosphate binding & 14 & $5.05 \times 10^{3}$ \\
\hline
\end{tabular}

B, downregulated

\begin{tabular}{llr}
\hline GOTERM_BP_FAT & GO:0010927 cellular component assembly involved in morphogenesis & 8 \\
GOTERM_BP_FAT & GO:0030030 cell projection organization & $9.34 \times 10^{4}$ \\
GOTERM_BP_FAT & GO:0007018 microtubule-based movement & $1.18 \times 10^{3}$ \\
GOTERM_BP_FAT & GO:0044782 cilium organization & 7 \\
GOTERM_BP_FAT & GO:0007017 microtubule-based process & $7.26 \times 10^{3}$ \\
GOTERM_CC_FAT & GO:0005929 cilium & $1.28 \times 10^{3}$ \\
GOTERM_CC_FAT & GO:0044441 ciliary part & $1.54 \times 10^{3}$ \\
GOTERM_CC_FAT & GO:0005930 axoneme & $2.75 \times 10^{7}$ \\
GOTERM_CC_FAT & GO:0097014 ciliary plasm & $1.00 \times 10^{3}$ \\
GOTERM_CC_FAT & GO:0030286 dynein complex & 16 \\
GOTERM_MF_FAT & GO:0003774 motor activity & 9 \\
GOTERM_MF_FAT & GO:0004024 alcohol dehydrogenase activity, zinc-dependent & 4 \\
GOTERM_MF_FAT & GO:0004022 alcohol dehydrogenase (NAD) activity & 4 \\
\end{tabular}

GO, Gene Ontology; DEG, differentially expressed gene; EEC, endometrioid endometrial carcinoma; ATP, adenosine triphosphate; NAD, nicotinamide adenine dinucleotide.

one significant module with a score $>4$ was extracted by the plugin MCODE, followed by further functional and pathway enrichment analyses (Fig. 3). This module was composed of 46 nodes and 970 edges, which were primarily involved in 'mitotic cell cycle process', 'cell cycle process' and 'mitotic cell cycle'. All the genes in this module were upregulated and closely interrelated.

Identification of the DEMs and analysis of miRNA-target regulatory network. Using GEO2R, a total of 57 DEMs were identified, including 51 upregulated DEMs and six downregulated DEMs (Fig. 4). From the results, hsa-miRNA(miR)-1290, hsa-miR-513a-5p, hsa-miR-1249, hsa-miR-9 and hsa-miR-557 were the top five upregulated DEMs, while hsa-miR-449a was the most downregulated DEM. The miRNA-target regulatory network was constructed using Cytoscape with the information from WebGestalt, containing 23 DEGs and 41 miRNAs (Fig. 4). From Fig. 4, miRNAs hsa-miR-9 and hsa-miR-15b were matched in the two results. Furthermore, their target genes forkhead box P1 (FOXP1) and cyclin E1 (CCNE1) were involved in the PPI network.

\section{Discussion}

EC is a common gynecological tumor, which is divided into two types. The degree of differentiation serves a crucial role in the prognosis of type I EC (EEC or EAC). EEC of G3 (poorly differentiated EEC) usually has a poor prognosis leading to metastasis in a short time. Therefore, it is important to examine the molecular mechanism of EEC differentiation 
Table II. KEGG pathway analysis of the DEGs associated with the degree of differentiation in EEC.

\begin{tabular}{|c|c|c|c|}
\hline Term & Count & P-value & Genes \\
\hline \multicolumn{4}{|l|}{ A, upregulated } \\
\hline cfa04110:Cell cycle & 9 & $1.55 \times 10^{8}$ & $\begin{array}{l}\text { CCNB1, CCNE1, CDC45, YWHAZ,MAD2L1, } \\
\text { BUB1, TTK, CDC25C, MCM4 }\end{array}$ \\
\hline cfa04114:Oocyte meiosis & 8 & $1.37 \times 10^{7}$ & $\begin{array}{l}\text { CCNE1, YWHAZ, MAD2L1, SGO1, BUB1, } \\
\text { FBXO5, AURKA, CDC25C }\end{array}$ \\
\hline cfa04914:Progesterone-mediated oocyte maturation & 4 & $4.62 \times 10^{3}$ & CCNB1, MAD2L1, BUB1, CDC25C \\
\hline cfa01130:Biosynthesis of antibiotics & 5 & $9.42 \times 10^{3}$ & PGK1, PAICS, PPAT, CBS, GLDC \\
\hline cfa00260:Glycine, serine and threonine metabolism & 3 & $1.03 \times 10^{2}$ & GAMT, CBS, GLDC \\
\hline cfa04115:p53 signaling pathway & 3 & $2.72 \times 10^{2}$ & CCNB1, CCNE1, RRM2 \\
\hline
\end{tabular}

B, downregulated

hsa00982:Drug metabolism-cytochrome P450

hsa00980:Metabolism of xenobiotics by cytochrome $\mathrm{P} 450$

hsa05204:Chemical carcinogenesis

hsa00350:Tyrosine metabolism

hsa00010:Glycolysis/Gluconeogenesis

hsa05016:Huntington's disease

\author{
3.06 $10^{3}$ GSTA3, ALDH1A3, ADH6, ADH1B \\ $3.89 \times 10^{3}$ GSTA3, ALDH1A3, ADH6, ADH1B \\ $4.84 \times 10^{3}$ GSTA3, ALDH1A3, ADH6, ADH1B \\ $9.92 \times 10^{3}$ ALDH1A3, ADH6, ADH1B \\ $33.38 \times 10^{2}$ ALDH1A3, ADH6, ADH1B \\ $44.95 \times 10^{2}$ DNAH9, DNAH12, CASP8, DNAH6
}

KEGG, Kyoto Encyclopedia of Genes and Genomes; DEGs, differentially expressed genes; EEC, endometrioid endometrial carcinoma; cfa, canis familiaris; hsa, homo sapiens.

and bioinformatics analysis of microarrays, facilitating the genetic study of EEC. In the present study, microarray datasets GSE17025, GSE24537 and GSE35784 were downloaded from the GEO database, and the difference between well-differentiated and poorly differentiated samples was analyzed. A total of 83 upregulated and 130 downregulated DEGs, together with 51 upregulated and six downregulated DEMs, were identified. In order to elucidate the category and pathway that the DEGs were involved in, GO and KEGG enrichment analyses were performed. Subsequently, the PPI network and subnetworks were constructed with the hub DEGs screened. A miRNA-target regulatory network was constructed with DEGs and their regulatory miRNAs. Subsequently, by combining the DEMs, miRNA-target regulatory network and PPI network, crucial DEGs were identified.

According to the results of the PPI network, TOP2A, KIF11, CCNB1 and BUB1 were identified as the foremost hub DEGs. They were all involved in one module extracted from the PPI network. DEGs in this module interacted closely with each other, and were primarily involved in 'mitotic cell cycle process' and 'cell cycle process'. From the GO and KEGG pathway analyses, TOP2A, KIF11, CCNB1 and BUB1 were all enriched in cell cycle-associated BP and pathways. hsa-miR-9 was identified as one of the top five upregulated DEMs; meanwhile, it was additionally involved in the miRNA-target regulatory network, as was hsa-miR-15b. Their respective target genes, the downregulated DEG FOXP1 and upregulated DEG CCNE1, were concurrently involved in the PPI network.

TOP2A encodes a DNA topoisomerase, an enzyme involved in DNA transcription and replication processes including chromosome condensation and chromatid separation. Thus, TOP2A serves a principal role in controlling the cell cycle and maintaining the integrity of the genome. It was reported that TOP2A was upregulated in numerous cancer types, including endometrial cancer $(3,4,15)$, pancreatic cancer $(16)$, prostate cancer (17) and breast cancer (18). In agreement with the present results, the increased TOP $2 \mathrm{~A}$ expression level indicated an association with more aggressive tumor behavior, including myometrial infiltration, and was associated with the prognosis in patients with EC (4,15). Moreover, TOP2A may be used as a biomarker in patients receiving adjuvant taxane-platinum regimens with moderate- to high-risk EC (3). The previous study (3) coincides with the present result that TOP2A was significantly upregulated in EC of G3 compared with EC of G1. In 2018, Pei et al (16) reported that the upregulation of TOP2A was observed in pancreatic cancer compared with non-tumor tissues, and significantly correlated with tumor metastasis and shorter survival. In a previous study (16), it was additionally revealed that a novel miR-139\TOP2A\ $\beta$-catenin axis drives the malignant progression of pancreatic cancer. In this axis, TOP2A induces cell proliferation and epithelial-mesenchymal transition (EMT) by activating the $\beta$-catenin signaling pathway, however, the process may be reversed by miR-139 (16). Zheng et al (18), demonstrated that the overexpression of TOP2A was associated with unfavorable biological behaviors of triple-negative breast cancer (TNBC), and TOP2A was confirmed as an independent prognostic indicator of 5-year disease-free survival. In prostate cancer, TOP2A was selected as a biomarker for early identification of patients with higher metastatic potential, thus these patients 


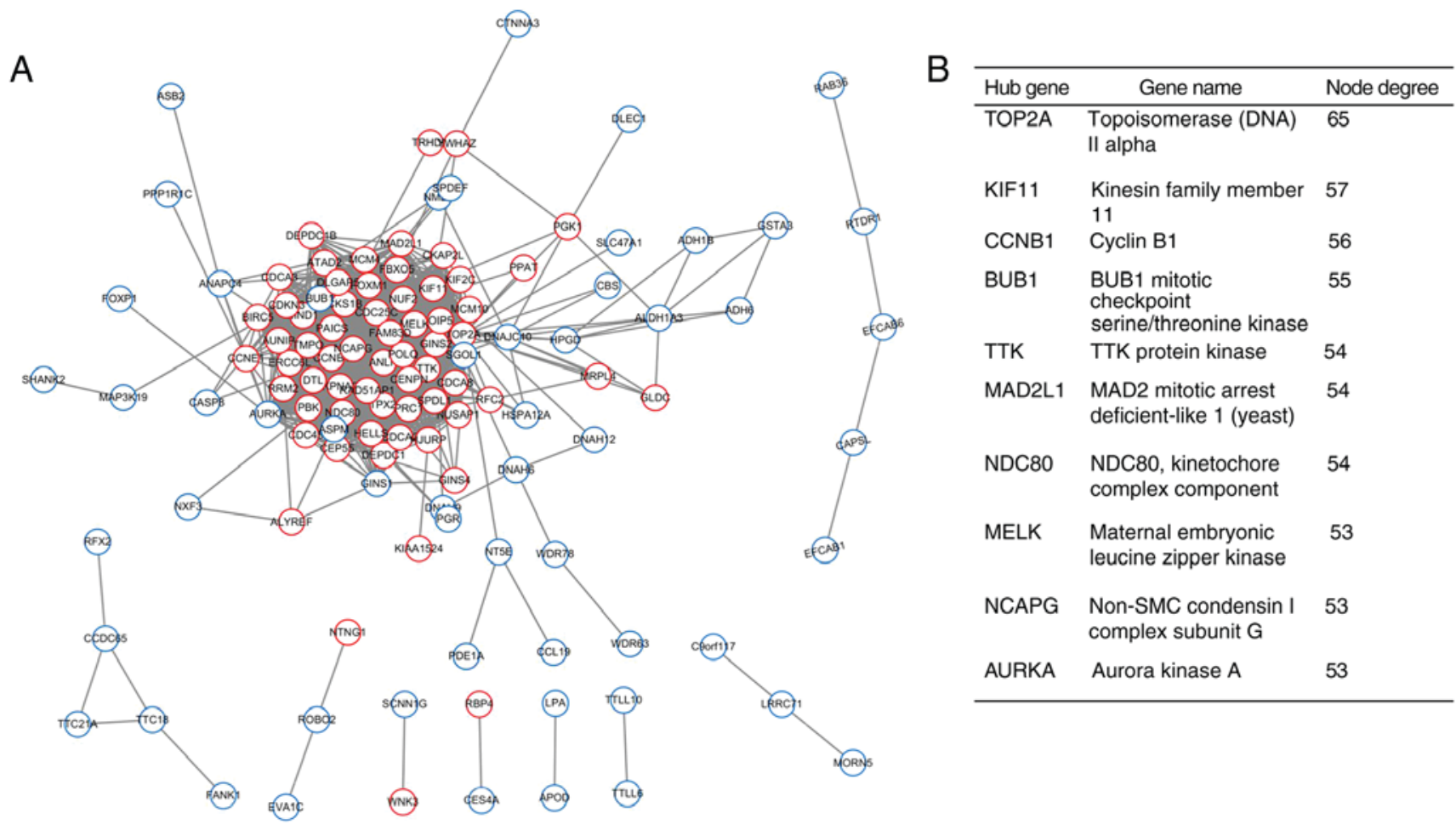

Figure 2. PPI network and hub genes. (A) PPI network of the DEGs. Red, upregulated hub genes; blue, downregulated hub genes. (B) Hub genes of the PPI network with higher node degrees. PPI, protein-protein interaction; DEGs, differentially expressed genes.

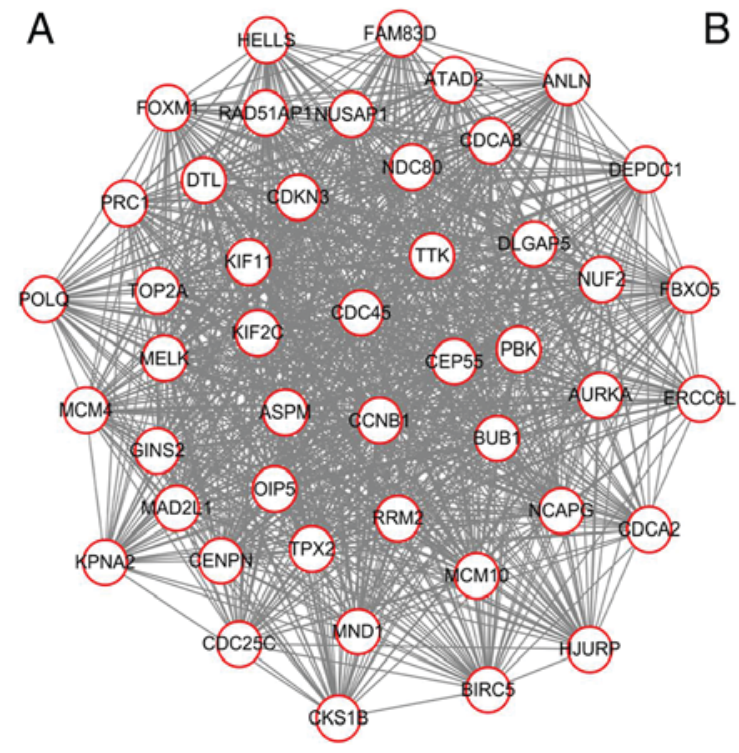

\begin{tabular}{|c|c|c|c|c|}
\hline Category & Term & Count & P-value & Genes \\
\hline GOTERM_BP_FAT & $\begin{array}{l}\text { GO:1903047 } \\
\text { mitotic cell cycle } \\
\text { process }\end{array}$ & 23 & $5.57 \mathrm{E}-23$ & $\begin{array}{l}\text { CENPN, GINS2, KIF11, FOXM1, NUF2, } \\
\text { TPX2, NUSAP1, TTK, BIRC5, AURKA, } \\
\text { NDC80, ANLN, CDC25C, CDKN3, } \\
\text { KIF2C, CDC45,CDCA8, MAD2L1, } \\
\text { NCAPG, BUB1, FBXO5, TOP2A, ASPM }\end{array}$ \\
\hline GOTERM_BP_FAT & $\begin{array}{l}\text { GO:0022402 } \\
\text { cell cycle process }\end{array}$ & 26 & 1.05E-21 & $\begin{array}{l}\text { PRC1, FOXM1, TTK, ANLN, AURKA, } \\
\text { KIF2C, CDC45, CDCA8, NCAPG, BUB1, } \\
\text { FBXO5, TOP2A, ASPM, CENPN, GINS2, } \\
\text { KIF11, DTL, NUF2, TPX2, MND1, } \\
\text { NUSAP1, BIRC5, NDC80, CDKN3, } \\
\text { CDC25C, MAD2L1 }\end{array}$ \\
\hline GOTERM_BP_FAT & $\begin{array}{l}\text { GO:0000278 } \\
\text { mitotic cell cycle }\end{array}$ & 23 & $1.51 \mathrm{E}-21$ & $\begin{array}{l}\text { CENPN, GINS2, KIF11, FOXM1, NUF2, } \\
\text { TPX2, NUSAP1, TTK, BIRC5, AURKA, } \\
\text { NDC80, ANLN, CDC25C, CDKN3, } \\
\text { KIF2C, CDC45, CDCA8, MAD2L1, } \\
\text { NCAPG, BUB1, FBXO5, TOP2A, ASPM }\end{array}$ \\
\hline KEGG_PATHWAY & $\begin{array}{l}\text { cfa04110: } \\
\text { cell cycle }\end{array}$ & 7 & 2.66E-08 & $\begin{array}{l}\text { CCNB1, CDC45, MAD2L1, BUB1, TTK, } \\
\text { CDC25C, MCM } 4\end{array}$ \\
\hline KEGG_PATHWAY & $\begin{array}{l}\text { cfa04114: } \\
\text { oocyte meiosis }\end{array}$ & 5 & 2.63E-05 & $\begin{array}{l}\text { MAD2L1, BUB1, FBXO5, AURKA, } \\
\text { CDC25C }\end{array}$ \\
\hline KEGG_PATHWAY & $\begin{array}{l}\text { cfa04914: } \\
\text { progesterone- } \\
\text { mediated } \\
\text { oocyte maturation }\end{array}$ & 4 & 3.99E-04 & CCNB1, MAD2L1, BUB1, CDC25C \\
\hline
\end{tabular}

Figure 3. Significant module and enrichment analyses. (A) Significant module of the PPI network. Red nodes denote the upregulated genes. (B) GO and KEGG enrichment analyzes of genes in the significant module. Only the top three BPs are presented. PPI, protein-protein interaction; GO, Gene Ontology; KEGG, Kyoto Encyclopedia of Genes and Genomes; BP, biological processes.

may receive timely adjuvant or neoadjuvant targeted therapy to improve prognosis (17). In summary, TOP2A is likely to have an important role in the genesis of poorly differentiated EC and may be used as an indicator of poor prognosis.

KIF11 is a motor kinesin required for the control of the accurate arrangement of microtubules and the separation of duplicated centrosomes during spindle formation. Overexpression of KIF11 is common in breast cancer, esophageal cancer, colorectal cancer and glioblastoma (19-21).
According to certain studies, the high expression level of KIF11 is significantly associated with poor prognosis among patients with non-small cell lung cancer (NSCLC) (22), oral cancer (23) and TNBC (24). Inhibition experiments were performed to examine the role of KIF11 in cancer cells. KIF11 was proven to be vital for proliferation and self-renewal in TNBC tumor cells (24). In vitro, knockdown of KIF11 via small interfering RNA resulted in a significant decrease in the percentage of cancer stem cells (CSCs) and mammosphere formation, with 
A

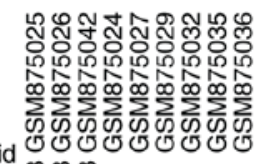

Grade

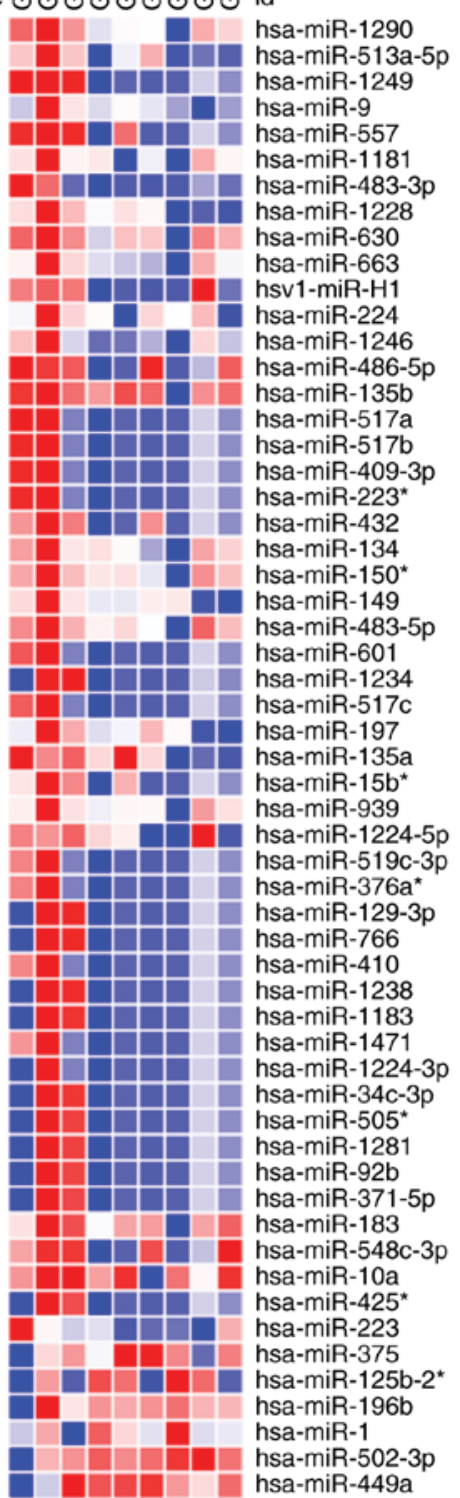

B
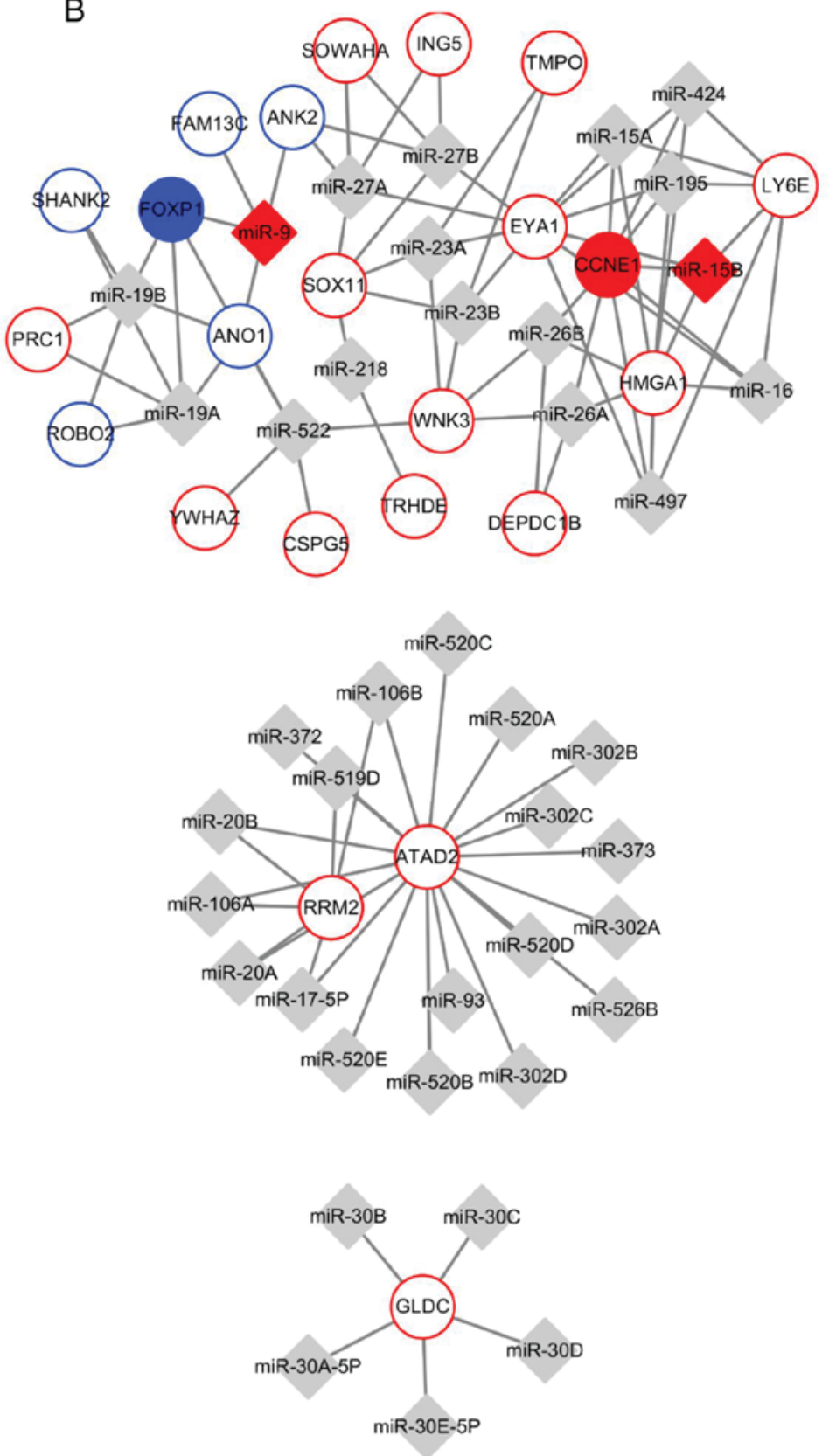

Figure 4. Heat map of the DEMs and miRNA-target regulatory network. (A) Heat map of the DEMs in GSE35784. Red, upregulated DEMs; blue, downregulated DEMs. (B) miRNA-target regulatory network constructed with DEGs and their regulatory miRNAs. Circular nodes represent DEGs and diamond-shaped nodes represent miRNAs. Red and blue circular nodes represent up and downregulated DEGs, respectively, among which the filled nodes represent DEGs additionally involved in the PPI network. Red diamond-shaped nodes represent upregulated miRNAs that match with the DEMs. DEMs, differentially expressed miRNAs; miRNA/miR, microRNA; DEGs, differentially expressed genes; PPI, protein-protein interaction.

additional cell cycle G2/M arrest, cell growth inhibition and apoptosis. In vivo, a KIF11 inhibitor exerted an inhibitory growth effect in docetaxel-resistant TNBC xenograft models. Similarly, knockdown of KIF11 or the application of KIF11 inhibitors suppressed cell proliferation, possibly through G2/M arrest, followed by the induction of apoptosis in oral cancer cells (23). In the study of Imai et al (20), knockdown of KIF11 inhibited mammosphere formation in esophageal and colorectal CSCs. Thus, the overexpression of KIF11 is associated with development and poor outcomes of cancer types, possibly by influencing the mammosphere formation of CSCs and cell cycle regulation.
BUB1 is the gene encoding the mitotic checkpoint serine/threonine kinase BUB1, a type of spindle assembly checkpoint protein kinase required for accurate chromosome segregation. It is important to maintain genome stability during mitosis and meiosis. An insufficiency of BUB1 expression triggers chromosomal instability, aneuploidy and premature senescence. An association between BUB1 expression and poor clinical prognosis was inferred in patients with breast cancer (25). The depletion of BUB1 suppressed the CSC potential of the MDA-MB-231 breast cancer cell line (25). Davidson et al (26) reported that in advanced-stage ovarian serous carcinoma, BUB1 was significantly coexpressed with 
aurora kinase A (AURKA). As a member of another family of mitotic serine/threonine kinases, AURKA is a hub gene in the significant module in the present study. Based on the study by Nyati et al (27), BUB1, as an integral component of transforming growth factor $\beta$ (TGF $\beta$ ) signaling, may promote the formation of TGFBRI/II receptor complex, which regulates downstream signaling pathways, including the mothers against decapentaplegic homolog, mitogen-activated protein kinase and phosphoinositide 3-kinase/RAC- $\alpha$ serine/threonine-protein kinase pathways, and mediate TGF $\beta$-dependent EMT, cell migration and invasion. All the aforementioned studies were in support of the facilitating effect of BUB1 on tumorigenesis and progression. However, in EC, it was declared that low expression of BUB1 was closely associated with poorly differentiated EC, which is contrary to the present results (28). However, another study investigating the expression of BUB1 in EC reported that BUB1 was upregulated in serous EC compared with EEC (29). As is known, the malignant potential of serous EC is higher compared with that of EEC. Thus, BUB1 is possibly positively associated with the malignancy of EC. In summary, the role of BUB1 in EC development remains undetermined

As a principal cell cycle regulator, $\mathrm{G} 2 /$ mitotic-specific cylin-B1 (the protein product of CCNB1) controls the G2/M transition by combining with cyclin-dependent kinase 1 to form a complex, which may promote progression to mitosis and amplify the cellular grow th rate. Therefore, the aberrant expression of CCNB1 contributes to tumorigenesis. The overexpression of CCNB1 has been observed in a multitude of tumors, including breast cancer, lung cancer, colorectal cancer and bladder cancer (30-32). Kim et al (32) demonstrated that FOXM1-CCNB1 signaling may be associated with the poor prognosis of non-muscle-invasive bladder cancer, and the expression of CCNB1 is able to predict the risk of recurrence in these patients. It was reported that inhibition or knockdown of CCNB1 suppressed cell proliferation, blocked cell cycle progression and induced cell apoptosis in colorectal cancer (31). The single nuecleotide polymorphisms (SNPs) of rs2069429 and rs2069433 in CCNB1 were verified to be associated with overall survival in NSCLC (30). Similarly, SNP rs2069433 in CCNB1 was revealed to be associated with the risk of EC (33). Furthermore, CCNB1, as a myc proto-oncogene downstream transcriptional target, was identified to be negatively correlated with the tumor suppressor progesterone receptor in EC (34). As mentioned above, CCNB1 may serve an important role in the genesis of EC and may be a powerful predictor of disease prognosis.

CCNE1 encodes the protein G1/S-specific cyclin-E1, which belongs to the highly conserved cyclin family, a cell cycle regulator. The protein forms a complex with cyclin-dependent kinase 2 and controls the $\mathrm{G} 1$ to $\mathrm{S}$ phase transition. The overexpression of CCNE1 may be an early event in tumorigenesis. In accordance with the present study, amplification of CCNE1 was reported to be significantly associated with high grade EEC and non-endometrioid EC (35). More notably, the expression level of CCNE1 in poorly differentiated EAC cell line was detected as higher compared with in well-differentiated EAC cell line (1.6 \pm 0.19 vs. $1.4 \pm 0.33)$ (1). In ovarian cancer, CCNE1 amplification confers worse survival and may be used as a potential therapeutic target (36). In lung adenocarcinoma (LUAD), the downregulation of CCNE1 may be induced by knockdown of homeobox C13 (HOXC13), leading to arrest at the G1 phase and subsequent inhibition of cell proliferation; whereas, miR-141 may exert the same effect by reducing the expression of HOXC13 and CCNE1 (37). Similarly, the inhibition of family with sequence similarity 83 member D may additionally suppress the proliferation of LUAD cells by interfering with the cell cycle by suppressing the expression of CCNE1 (38). Notably, according to the study from Krivega et al (39), CCNE1 exerts a crucial role in balancing totipotency and differentiation in human embryonic cells, which means that CCNE1 possesses the potential of regulating cell differentiation. In summary, it was confirmed that CCNE1 is upregulated in poorly differentiated EEC and is important in cell differentiation.

FOXP1, a P subfamily of forkhead box, is an estrogenresponsive transcription factor and is implicated in cellular differentiation and proliferation. Previous studies (2,40-43) suggested that FOXP1 may be a tumor suppressor gene in breast cancer, prostate cancer, epithelial ovarian cancer and EC. A meta-analysis of 22 articles that examined nine tumor types and included 2,468 patients reported that reduced FOXP1 expression was associated with worse survival in patients with solid tumors (40). It was reported that the activation of FOXP1 may inhibit tumor proliferation in epithelial ovarian cancer (41). Conversely, the downregulation of FOXP1 expression regulated by miR-504 triggered tumorigenesis in glioma (42). Consistent with the present study, lower FOXP1 expression in the nucleus and cytoplasm of G3 EEC was detected when compared with G1 and G2 EEC in a study conducted by Mizunuma et al (2). This previous study also reported that FOXP1 acted as a tumor suppressor in EEC through the GTPase KRAS pathway. Furthermore, Giatromanolaki et al (43) demonstrated that the loss of FOXP1 expression resulted in a slightly worse outcome in early EC. According to the aforementioned literature, the inverse effect of FOXP1 was assured in the process of tumorigenesis and poor differentiation of EEC.

The microRNA miR-9 is a small, non-coding RNA involved in gene regulation. As demonstrated in Fig. 4, miR-9 was one of the top upregulated DEMs in the heatmap and interacted with a number of downregulated DEGs in the miRNA-target regulatory network. In accordance with the present results, Torres et al (5) demonstrated that the expression level of miR-9 was lower in G1 samples compared with G2 and G3 samples of EEC. Additionally, miR-9 expression was reported to be upregulated in numerous cancer types, including colorectal cancer, breast cancer and lung cancer. As an example, miR-9 was upregulated in NSCLC, and functioned as the core of TGF- $\beta 1$-induced cell invasion and adhesion by targeting SOX7 (44). More notably, the upregulation of miR-9 and subsequent downregulation of its target, Cadherin-1, may induce EMT in NSCLC cells by regulating TGF- $\beta 1$ signaling (45). Furthermore, significant overexpression of miR-9 was observed in breast cancer tissues and cell lines, and miR-9 may promote the proliferation, migration and invasion of breast cancer cells by downregulating FOXO1 (46). As aforementioned, miR-9 may promote tumor progression by downregulating the target tumor suppressors. 
Principal genes were identified that may be determinants of the carcinogenesis of poorly differentiated EC, which may facilitate the investigation of the potential molecular mechanism. These genes may additionally help to identify candidate biomarkers and novel therapeutic targets of poorly differentiated EC. However, there are limitations of the present study. Among all the genes identified, only the genes TOP2A, CCNE1, FOXP1 and miR-9 have already been verified to be differentially expressed between G1 and G3 EC samples in previous studies; however, the remaining genes have not been studied in this regard. Similarly, overexpression of TOP2A has been demonstrated to be associated with the poor prognosis of EC in previous studies $(4,15)$, while the significance of prognostic evaluation of the remaining genes has been proven in other cancer types, although not in EC. Therefore, further laboratory tests of clinical samples are essential to confirm the present findings. Additionally, whether these genes may be used as biomarkers and therapeutic targets requires further experimental verification.

In conclusion, by comparing the G3 and G1 EEC samples, a total of 213 DEGs, including 83 upregulated and 130 downregulated DEGs, were identified; a total of 57 DEMs including 51 upregulated DEMs and six downregulated DEMs, were identified. Based on the PPI network, TOP2A, KIF11, CCNB1 and BUB1 were the main hub genes. According to the enrichment analysis of DEGs of the significant modules, they were primarily associated with 'mitotic cell cycle process', 'cell cycle process' and 'mitotic cell cycle'. Based on the analysis of DEMs and the miRNA-target regulatory network, miR-9 may be the most important upregulated DEM, and the DEGs FOXP1 and CCNE1 may serve vital roles in the differentiation of EC. These identified principal genes may help to identify the potential molecular mechanism of tumorigenesis and additionally, candidate biomarkers and novel targeted therapy for poorly differentiated EC. However, further laboratory tests of clinical samples or laboratory research are required to verify the reliability of the results in the present study.

\section{Acknowledgements}

The present study was supported by grants from the Natural Science Foundation of China (grant nos. 81572568 and 81272863).

\section{Funding}

The present study was supported by grants from the Natural Science Foundation of China (grant nos. 81572568 and 81272863).

\section{Availability of data and materials}

The datasets analysed during the current study are available in the Gene Expression Omnibus repository (http://www.ncbi. nlm.nih.gov/geo/).

\section{Authors' contributions}

YZ, YW and FX conceived and designed the study. YZ, $\mathrm{MD}, \mathrm{KZ}$ and $\mathrm{WT}$ performed the experiments. YZ wrote the manuscript. YW and FX revised and edited the manuscript. All authors read and approved the final manuscript.

\section{Ethics approval and consent to participate}

Not applicable.

\section{Consent for publication}

Not applicable.

\section{Competing interests}

The authors declare that they have no competing interests.

\section{References}

1. Nowakowska M, Matysiak-Burzyńska Z, Kowalska K, Płuciennik E, Domińska K and Piastowska-Ciesielska AW: Angiotensin II promotes endometrial cancer cell survival. Oncol Rep 36: 1101-1110, 2016.

2. Mizunuma M, Yokoyama Y, Futagami M, Horie K, Watanabe J and Mizunuma H: FOXP1 forkhead transcription factor is associated with the pathogenesis of endometrial cancer. Heliyon 2: e00116, 2016

3. Ito F, Furukawa $\mathrm{N}$ and Nakai T: Evaluation of TOP2A as a predictive marker for endometrial cancer with taxane-containing adjuvant chemotherapy. Int J Gynecol Cancer 26: 325-330, 2016.

4. Supernat A, Łapińska-Szumczyk S, Majewska H, Gulczyński J, Biernat W, Wydra D and Zaczek AJ: A multimarker qPCR platform for the characterisation of endometrial cancer. Oncol Rep 31: 1003-1013, 2014.

5. Torres A, Torres K, Pesci A, Ceccaroni M, Paszkowski T, Cassandrini P, Zamboni G and Maciejewski R: Diagnostic and prognostic significance of miRNA signatures in tissues and plasma of endometrioid endometrial carcinoma patients. Int J Cancer 132: 1633-1645, 2013

6. Koh WJ, Greer BE, Abu-Rustum NR, Apte SM, Campos SM, Chan J, Cho KR, Cohn D, Crispens MA, Dupont N, et al: Uterine neoplasms, version 1.2017. 2016 National Comprehensive Cancer Network Inc., 2017.

7. Kaloglu S, Guraslan H, Tekirdag AI, Dagdeviren H and Kaya C: Relation of preoperative thrombocytosis between tumor stage and grade in patients with endometrial cancer. Eurasian J Med 46: 164-168, 2014.

8. Colombo N, Creutzberg C, Amant F, Bosse T, González-Martín A, Ledermann J, Marth C, Nout R, Querleu D, Mirza MR, et al: ESMO-ESGO-ESTRO consensus conference on endometrial cancer: Diagnosis, treatment and follow-up. Radiother Oncol 117: 559-581, 2015.

9. Mou T, Zhu D, Wei X, Li T, Zheng D, Pu J, Guo Z and Wu Z: Identification and interaction analysis of key genes and microRNAs in hepatocellular carcinoma by bioinformatics analysis. World J Surg Oncol 15: 63, 2017.

10. Bastos HP, Tavares B, Pesquita C, Faria D and Couto FM: Application of gene ontology to gene identification. Methods Mol Biol 760: 141-157, 2011.

11. Kanehisa M, Sato Y, Kawashima M, Furumichi M and Tanabe M: KEGG as a reference resource for gene and protein annotation. Nucleic Acids Res 44: D457-D462, 2016.

12. Huang da W, Sherman BT and Lempicki RA: Systematic and integrative analysis of large gene lists using DAVID bioinformatics resources. Nat Protoc 4: 44-57, 2009.

13. Szklarczyk D, Franceschini A, Wyder S, Forslund K, Heller D, Huerta-Cepas J, Simonovic M, Roth A, Santos A, Tsafou KP, et al: STRING v10: Protein-protein interaction networks, integrated over the tree of life. Nucleic Acids Res 43 (Database Issue): D447-D452, 2015.

14. Su G, Morris JH, Demchak B and Bader GD: Biological network exploration with Cytoscape 3. Curr Protoc Bioinformatics 47: 8.13.1-24, 2014.

15. Supernat A, Lapińska-Szumczyk S, Majewska H, Gulczyński J, Biernat W, Wydra D and Zaczek AJ: Tumor heterogeneity at protein level as an independent prognostic factor in endometrial cancer. Transl Oncol 7: 613-619, 2014. 
16. Pei YF, Yin XM and Liu XQ: TOP2A induces malignant character of pancreatic cancer through activating $\beta$-catenin signaling pathway. Biochim Biophys Acta 1864: 197-207, 2018.

17. Labbé DP, Sweeney CJ, Brown M, Galbo P, Rosario S Wadosky KM, Ku SY, Sjöström M, Alshalalfa M, Erho N, et al: TOP2A and EZH2 provide early detection of an aggressive prostate cancer subgroup. Clin Cancer Res 23: 7072-7083, 2017.

18. Zheng H, Li X, Chen C, Chen J, Sun J, Sun S, Jin L, Li J, Sun S and $\mathrm{Wu} \mathrm{X}$ : Quantum dot-based immunofluorescent imaging and quantitative detection of TOP2A and prognostic value in triple-negative breast cancer. Int J Nanomedicine 11: 5519-5529, 2016.

19. Pei YY, Li GC, Ran J and Wei FX: Kinesin family member 11 contributes to the progression and prognosis of human breast cancer. Oncol Lett 14: 6618-6626, 2017.

20. Imai T, Oue N, Sentani K, Sakamoto N, Uraoka N, Egi H, Hinoi T, Ohdan H, Yoshida K and Yasui W: KIF11 is required for spheroid formation by oesophageal and colorectal cancer cells. Anticancer Res 37: 47-55, 2017.

21. Venere M, Horbinski C, Crish JF, Jin X, Vasanji A, Major J, Burrows AC, Chang C, Prokop J, Wu Q, et al: The mitotic kinesin KIF11 is a driver of invasion, proliferation, and self-renewal in glioblastoma. Sci Transl Med 7: 304ra143, 2015.

22. Schneider MA, Christopoulos P, Muley T, Warth A, Klingmueller U, Thomas M, Herth FJ, Dienemann H, Mueller NS Theis $\mathrm{F}$ and Meister M: AURKA, DLGAP5, TPX2, KIF11 and CKAP5: Five specific mitosis-associated genes correlate with poor prognosis for non-small cell lung cancer patients. Int J Oncol 50: 365-372, 2017

23. Daigo K, Takano A, Thang PM, Yoshitake Y, Shinohara M, Tohnai I, Murakami Y, Maegawa J and Daigo Y: Characterization of KIF11 as a novel prognostic biomarker and therapeutic target for oral cancer. Int J Oncol 52: 155-165, 2018

24. Jiang M, Zhuang H, Xia R, Gan L, Wu Y, Ma J, Sun Y and Zhuang Z: KIF11 is required for proliferation and self-renewal of docetaxel resistant triple negative breast cancer cells. Oncotarget 8: 92106-92118, 2017.

25. Han JY, Han YK, Park GY, Kim SD, Kim JS, Jo WS and Lee CG: Bub1 is required for maintaining cancer stem cells in breast cancer cell lines. Sci Rep 5: 15993, 2015.

26. Davidson B, Nymoen DA, Elgaaen BV, Staff AC, Tropé CG, Kaern J, Reich R and Falkenthal TE: BUB1 mRNA is significantly co-expressed with AURKA and AURKB mRNA in advanced-stage ovarian serous carcinoma. Virchows Arch 464: 701-707, 2014.

27. Nyati S, Schinske-Sebolt K, Pitchiaya S, Chekhovskiy K, Chator A, Chaudhry N, Dosch J, Van Dort ME, Varambally S, Kumar-Sinha C, et al: The kinase activity of the Ser/Thr kinase BUB1 promotes TGF-beta signaling. Sci Signal 8: ra1, 2015.

28. Li L, Xu DB, Zhao XL and Hao TY: Combination analysis of Bub1 and Mad2 expression in endometrial cancer: Act as a prognostic factor in endometrial cancer. Arch Gynecol Obstet 288: 155-165, 2013.

29. Chen Y, Yao Y, Zhang L, Li X, Wang Y, Zhao L, Wang J, Wang G, Shen D, Wei L and Zhao J: cDNA microarray analysis and immunohistochemistry reveal a distinct molecular phenotype in serous endometrial cancer compared to endometrioid endometrial cancer. Exp Mol Pathol 91: 373-384, 2011.

30. Liu D, Xu W, Ding X, Yang Y, Su B and Fei K: Polymorphisms of CCNB1 associated with the clinical outcomes of platinum-based chemotherapy in Chinese NSCLC patients. J Cancer 8: 3785-3794, 2017

31. Fang Y, Yu H, Liang X, Xu J and Cai X: Chk1-induced CCNB1 overexpression promotes cell proliferation and tumor growth in human colorectal cancer. Cancer Biol Ther 15: 1268-1279, 2014.
32. Kim SK, Roh YG, Park K, Kang TH, Kim WJ, Lee JS, Leem SH and Chu IS: Expression signature defined by FOXM1-CCNB1 activation predicts disease recurrence in non-muscle-invasive bladder cancer. Clin Cancer Res 20: 3233-3243, 2014.

33. Cai H, Xiang YB, Qu S, Long J, Cai Q, Gao J, Zheng W and Shu XO: Association of genetic polymorphisms in cell-cycle control genes and susceptibility to endometrial cancer among Chinese women. Am J Epidemiol 173: 1263-1271, 2011

34. Kavlashvili T, Jia Y, Dai D, Meng X, Thiel KW, Leslie KK and Yang S: Inverse relationship between progesterone receptor and Myc in endometrial cancer. PLoS One 11: e0148912, 2016.

35. Noske A, Brandt S, Valtcheva N, Wagner U, Zhong Q, Bellini E, Fink D, Obermann EC, Moch $\mathrm{H}$ and Wild PJ: Detection of CCNE1/URI (19q12) amplification by in situ hybridisation is common in high grade and type II endometrial cancer. Oncotarget 8: 14794-14805, 2017.

36. Nakayama N, Nakayama K, Shamima Y, Ishikawa M, Katagiri A, Iida K and Miyazaki K: Gene amplification CCNE1 is related to poor survival and potential therapeutic target in ovarian cancer. Cancer 116: 2621-2634, 2010

37. Yao Y, Luo J, Sun Q, Xu T, Sun S, Chen M, Lin X, Qian Q, Zhang Y, Cao L, et al: HOXC13 promotes proliferation of lung adenocarcinoma via modulation of CCND1 and CCNE1. Am J Cancer Res 7: 1820-1834, 2017.

38. Shi R, Sun J, Sun Q, Zhang Q, Xia W, Dong G, Wang A, Jiang F and $\mathrm{Xu}$ L: Upregulation of FAM83D promotes malignant phenotypes of lung adenocarcinoma by regulating cell cycle. Am J Cancer Res 6: 2587-2598, 2016

39. Krivega MV, Geens M, Heindryckx B, Santos-Ribeiro S, Tournaye $\mathrm{H}$ and Van de Velde $\mathrm{H}$ : Cyclin E1 plays a key role in balancing between totipotency and differentiation in human embryonic cells. Mol Hum Reprod 21: 942-956, 2015.

40. Xiao J, He B, Zou Y, Chen X, Lu X, Xie M, Li W, He S, You S and Chen Q: Prognostic value of decreased FOXP1 protein expression in various tumors: A systematic review and meta-analysis. Sci Rep 6: 30437, 2016.

41. Hu Z, Zhu L, Tan M, Cai M, Deng L, Yu G, Liu D, Liu J and Lin B: The expression and correlation between the transcription factor FOXP1 and estrogen receptors in epithelial ovarian cancer. Biochimie 109: 42-48, 2015.

42. Cui R, Guan Y, Sun C, Chen L, Bao Y, Li G, Qiu B, Meng X, Pang $\mathrm{C}$ and Wang Y: A tumor-suppressive microRNA, miR-504, inhibits cell proliferation and promotes apoptosis by targeting FOXP1 in human glioma. Cancer Lett 374: 1-11, 2016.

43. Giatromanolaki A, Koukourakis MI, Sivridis E, Gatter KC, Harris AL and Banham AH: Loss of expression and nuclear/cytoplasmic localization of the FOXP1 forkhead transcription factor are common events in early endometrial cancer: Relationship with estrogen receptors and HIF-1alpha expression. Mod Pathol 19: 9-16, 2006.

44. Han L, Wang W, Ding W and Zhang L: MiR-9 is involved in TGF- $\beta 1$-induced lung cancer cell invasion and adhesion by targeting SOX7. J Cell Mol Med 21: 2000-2008, 2017.

45. Wang $\mathrm{H}, \mathrm{Wu} \mathrm{Q}$, Zhang $\mathrm{Y}$, Zhang $\mathrm{HN}$, Wang YB and Wang W: TGF- $\beta 1$-induced epithelial-mesenchymal transition in lung cancer cells involves upregulation of miR-9 and downregulation of its target, E-cadherin. Cell Mol Biol Lett 22: 22, 2017.

46. Liu DZ, Chang B, Li XD, Zhang QH and Zou YH: MicroRNA-9 promotes the proliferation, migration, and invasion of breast cancer cells via down-regulating FOXO1. Clin Transl Oncol 19: 1133-1140, 2017. 